\title{
Efficacy of Mupirocin Ointment in Eradication of Staphylococcus aureus Nasal Carriage in Intensive Care Unit Staff and Patients
}

\author{
Abolfazl Khandandel (PhD \\ Candidate) \\ Department of Microbiology, $5^{\text {th }}$ Azar \\ Hospital, Golstan University of \\ Medical Sciences, Gorgan, Iran \\ Ania Ahani Azari (PhD) \\ Department of Microbiology, Islamic \\ Azad University, Gorgan branch, \\ Gorgan, Iran \\ Ailar Jamalli (PhD) \\ Laboratory Sciences Research Center, \\ Golestan University of Medical \\ Sciences, Gorgan, Iran \\ Ezzat Allah Ghaemi (PhD) \\ Department of Microbiology, Faculty \\ of Medicine, Golestan University of \\ Medical Sciences, Gorgan, Iran \\ Corresponding author: Abolfazl \\ Khandandel \\ Tel: +98-174420651 \\ Email: ab.khandandel@gmail.com \\ Address: Department of \\ Microbiology, Faculty of Medicine, \\ Golestan University of Medical \\ Sciences, Gorgan, Iran \\ Received : 29 Jan 2018 \\ Revised: 02 Apr 2018 \\ Accepted: 08 Apr 2018

\begin{abstract}
Abolfazl Khandandel https://orcid.org/0000-00027866-2296

Aylar Jamali http://orcid.org/0000-0002-4612-8144 Ezzat Allah Ghaemi http://orcid.org/0000-0001-
\end{abstract} \\ $7727-7452$
}

\section{ABSTRACT}

Background and Objectives: Staphylococcus aureus is one of the most common causes of morbidity and mortality among intensive care unit (ICU) patients. Nasal carriage is one of the main routs of $S$. aureus transmission between hospital personnel and patients. The objective of this study was to evaluate the efficacy of mupirocin ointment in eradication of nasal carriage of $S$. aureus in the ICU staff and patients of Panje-Azar hospital in Gorgan, Iran.

Methods: In the first three months of the study (January to March), the prevalence of S. aureus among ICU patients was determined by routine microbiological and biochemical testing. Nasal samples were taken from ICU staff and all patients recently admitted to the ICU. Mupirocin nasal ointment (2\%) was applied for treatment of $S$. aureus nasal carriers. Posttreatment sampling was done after five weeks. During the next three months, the presence of S. aureus and rate of resistance to methicillin was evaluated in new patients admitted to the ICU using the method used previously.

Results: 0f 60 samples from the ICU staff, seven (11.7\%) samples were positive for $S$. aureus. Moreover, of 240 samples from the ICU patients, two samples were found as $S$. aureus-positive. Of the nine $S$. aureus-positive isolates, only two (22.2\%) were methicillinresistant S. aureus (NRSA). In the pre-intervention sampling, only five samples (2.0\%) were identified as $S$. aureus, two of which were MRSA. However, treatment with mupirocin ointment eradicated nasal carriage of $S$. aureus and no isolate was found after the intervention.

Conclusion: 0ur finding showed that mupirocin nasal ointment is highly effective in eradication of $S$. aureus nasal carriage and subsequently contribute to reduction in frequency of nosocomial infections in the ICU.

Keywords: Intensive Care Units, Mupirocin, Nasal, Staphylococcus aureus.

This paper should be cited as: Khandandel A, Ahani Azari A, Jamali A, Ghaemi EA[Efficacy of Mupirocin Ointment in Eradication of Staphylococcus aureus Nasal Carriage in Intensive Care Unit Staff and Patients]. mljgoums. 2018; $12(3): 12-16$ 


\section{INTRODUCTION}

Hospital-acquired infection is a global health problem associated with numerous factors. The etiological agents of these infections vary widely among different countries, but since 1980s, gram-positive bacteria especially Staphylococcus aureus have been considered as the main causative agent for nosocomial infections (1). The habitat of $S$. aureus is the anterior nasal canal and about $20 \%$ of the human population is thought to be carriers of this microorganism (2). The colonization rate of $S$. aureus in hospital staff is relatively higher than that in other individuals (3). These individuals are in constant contact with patients in different wards, which might consequently affect the progression of infection in the patients (1). The pathogenesis of $S$. aureus and its ability to acquire antimicrobial resistance have made this organism a major problem for hospitals and medical staff (3). S. aureus causes a wide range of infections including bacteremia, septicemia, pneumonia as well as skin, soft tissue and bone infections (4). Mupirocin is an ointment used for controlling staphylococcal infections in healthy carriers by inhibiting synthesis of isoleucyl-tRNA in the bacteria (5). Twice daily administration of mupirocin nasal ointment (2\%) for five days is the most effective treatment for methicillin-resistant $S$. aureus (MRSA) carriage. MRSA strains are important nosocomial pathogens that can cause potentially deadly diseases. The treatment of infections caused by MRSA strains has been challenging because of multiple-drug resistance (6). Although vancomycin has been known as the antibiotic of choice for the treatment of infections caused by MRSA strains, studies from different parts of the world have recently reported the emergence of vancomycin-intermediate $S$. aureus (VISA) strains. The pathogenesis of MRSA and its increasing rate of resistance to antibiotics might prolong hospital stay, distress the patients and increase treatment costs. Hence, it is essential to take necessary measures for the control and prevention of MRSA infections (7). Since these microorganisms can easily spread in the hospital environment through respiratory secretions and contaminated hands, the most effective method of prevention is screening of nasal carriers among medical staff. Presence of S. aureus in the intensive care units (ICUs) is of great importance due to use of invasive devices, prolonged hospital stay, weakened immune system, and frequent consumption of antibiotics (1). This study aimed to determine the efficiency of mupirocin ointment in eradication of $S$. aureus nasal carriage among the ICU staff and patients of Panje-azar hospital in Gorgan, Iran.

\section{MATERIAL AND METHODS}

This interventional study was performed on ICU staff and patients of Panjeazar teaching hospital during January-October 2014. The study received approval from the ethics committee of Golestan University of Medical Sciences (code: 509299302188). After obtaining necessary permission from the hospital authorities and written consent from the staff and family of patients, microbial culture of all samples from ICU patients were examined in the first three months of the study (January to March). Overall, 180 clinical samples were collected and cultured from 120 patients within 48 hours of admission to the hospital. Demographic data were recorded and studied for each patient. Samples found positive for $S$. aureus were recorded. S. aureus strains were detected by culture on mannitol salt agar and blood agar as well as biochemical testing according to standard guidelines (8). In the next two months (April and May), sampling was done with sterile nasal swaps from 60 personnel working in the hospital ICU and new patients admitted in the last 24 hours. Positive cultures for $S$. aureus were treated with mupirocin ointment (2\%) for five days according to the instructions (5). To ensure about the eradication of $S$. aureus carriage, sampling was done after five weeks from the individuals treated with mupirocin and the patients who were admitted to the hospital since April 2014. Methicillin resistance was examined by disk diffusion method using cefoxitin disk $(30 \mu \mathrm{g}$, Himedia Co.) according to the Clinical and Laboratory Standards Institute guidelines (9) and using polymerase chain reaction (PCR) for presence of mecA gene as described by Louie et al (10). The following MecA primer sequences (CinnaGen Co., Iran) were used for the PCR experiment: Forward:

TCCAGATTACAACTTCACCAGG, Reverse: CCACTTCATATCTTGTAACG. 
In the next three months (June to August), all cultures requested by the physician for the 120 ICU patients were examined and the results were recorded. The prevalence of $S$. aureus was determined in different periods and was later compared using chi-square test. P-values less than 0.05 were considered as statistically significant.

\section{RESULTS}

The most common reasons for hospital admission were car accidents $(48.3 \%)$ and falling accident (10\%). Level of consciousness in most patients was between 6 and 10 based on the Glascow Coma Scale. All patients were using urinary and venous catheters (Table1). Of 180 samples collected, 97 samples (53.9\%) were culture-positive, which were mostly related to chest tubes (40 cases) and blood samples (20 cases) (Table 2). Coagulasenegative staphylococci (16 cases), enterococci (15 cases), Escherichia (11 cases) and yeast (11 cases) were the most common organisms isolated. Of the 180 cultured samples from ICU patients in the first stage, S. aureus was isolated from five cases $(2.8 \%, 2$ men and 3 women), two of which were identified as MRSA (Figure 1). One case was an 18-yearold woman who was admitted to the ICU because of suicide attempt and died after 23 days. The second case was a 23-year-old woman who was hospitalized in the ICU due to a car accident (head injury) and died after 18 days. In this case, three samples were cultured and $S$. aureus was isolated in the sputum sample taken on the second day of hospitalization. The third case was a 29-yearold man who was admitted to the hospital due to leg fracture and was discharged after 11 days. For this patient, three blood samples were taken and cultured, and S. aureus was isolated on the fourth day. The next case was a 50-year-old woman who was hospitalized due to cardiac arrest and was discharged after 20 days. During her stay, four samples were cultured and $S$. aureus was isolated from the sputum culture on the tenth day. The fifth case was a 62-year-old man who was admitted to the hospital due to a falling accident, and was discharged after 10 days. In this case, two samples were cultured and MRSA was isolated from the chest tube sample taken on the tenth day.

Of the 60 nasal swab samples taken from the ICU staff, S. aureus was isolated only in seven samples $(11.7 \%)$, two of which $(22.2 \%)$ were identified as MRSA. Of the 120 nasal samples taken from the patients at admission, only two cases $(1.6 \%)$ were identified as $S$. aureus but none was MRSA strain. Treatment with mupirocin eradicated $S$. aureus in all five carriers, as the post-treatment culture from the nasal carriers was negative after five weeks. However, the post-treatment culture was not performed for two patients because they had been discharged before the end of the fifth week. In the next stage of the study, the samples were taken from the patients three months after the intervention. Overall, 146 clinical samples (85 from men) were cultured and examined, which were mostly related to blood (11 specimens), urine (10 specimens) and chest tube (6 specimens) (Table 2). The culture was positive for 35 cases $(23.97 \%)$. Enterococcus, couagulase-negative Staphylococcus and yeast were the most common isolated microorganisms from the ICU patients. S. aureus was not isolated from any of the samples. There was no significant difference between the pre- and postintervention characteristics of the patients in the two groups. As shown in table 2, the treatment with mupirocin significantly reduced the frequency of Staphylococcus.

Figure1- Results of PCR for presence of MRSA strains. Column M: 1 Kb DNA ladder, C-: negative control (no DNA), C+: positive control, Columns 1-5: samples

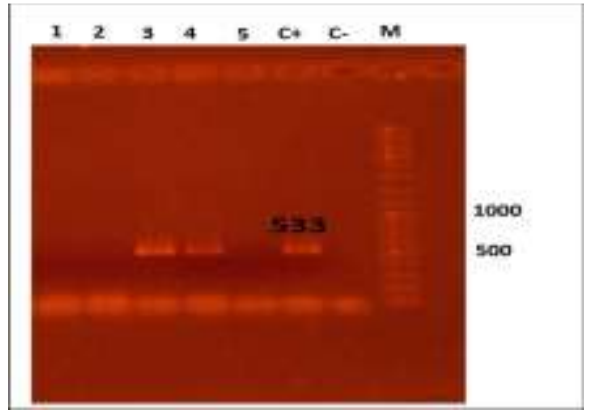


15/ Khandandel and colleagues

Table 1- Characteristics of the subjects before and after the intervention

\begin{tabular}{cccc}
\hline Characteristic & Before intervention & After intervention & P-value \\
\hline Number of patients & $\mathbf{1 2 0}$ & $\mathbf{1 2 0}$ & $\mathbf{0 . 9}$ \\
Mean age (years) & $\mathbf{4 5 . 5 \pm 2 0 . 3}$ & $\mathbf{4 4 . 1} \pm \mathbf{2 3 . 4}$ & $\mathbf{0 . 7}$ \\
Number of males & $\mathbf{7 3}$ patients $(\mathbf{6 0 . 8 \%}$ & $\mathbf{8 5}$ patients $(\mathbf{7 0 . 8 \%})$ & $\mathbf{0 . 0 7}$ \\
Average level of consciousness $(\mathbf{6 - 1 0})$ & $\mathbf{5 3}$ patients & $\mathbf{6 6}$ patients & $\mathbf{0 . 2}$ \\
Use of invasive devices and prostheses & & & \\
NG tube & $\mathbf{8 1}$ & $\mathbf{8 3}$ & $\mathbf{0 . 2}$ \\
Ventilator & $\mathbf{8 1}$ & $\mathbf{8 3}$ & $\mathbf{0 . 4}$ \\
Endotracheal tube & $\mathbf{8 1}$ & $\mathbf{8 3}$ & $\mathbf{0 . 5}$ \\
\hline
\end{tabular}

Table 2- Efficacy of mupirocin ointment in eradication of $S$. aureus nasal carriage in 120 ICU patients

\begin{tabular}{|c|c|c|c|}
\hline Characteristic & Before the intervention & After the intervention & P-value \\
\hline $\begin{array}{c}\text { Number of patients } \\
\text { Frequency of specimens }\end{array}$ & 120 & 120 & $\begin{array}{c}0.9 \\
0.006\end{array}$ \\
\hline Urine & 14 & 10 & \\
\hline Blood & 20 & 11 & \\
\hline NG tube & 40 & 6 & \\
\hline Others & 23 & 8 & \\
\hline Frequency of positive cultures & $97(54 \%)$ & $35(24 \%)$ & 0.002 \\
\hline Frequency of $S$. aureus & $5(4.2 \%)$ & $\mathbf{0}$ & 0.002 \\
\hline \multirow{2}{*}{\multicolumn{4}{|c|}{ Other bacteria }} \\
\hline & & & \\
\hline $\begin{array}{c}\text { Coagulase-negative } \\
\text { Staphylococcus }\end{array}$ & 16 & 6 & \\
\hline Yeast & 11 & 6 & \\
\hline Escherichia & 11 & 5 & \\
\hline Streptococcus sp. & 8 & 5 & \\
\hline Enterobacter & 9 & 3 & \\
\hline Others & 22 & 2 & \\
\hline Total & 92 & 35 & \\
\hline
\end{tabular}

\section{DISCUSSION}

In the present study, the initial frequency of $S$. aureus nasal carriage was $11.7 \%$ among the ICU staff, $3.3 \%$ of which were identified as MRSA strain. In study of Rahimi-Alang et al. on healthcare workers in Gorgan, the frequency of $S$. aureus and MRSA nasal carriage was $24 \%$ and $3 \%$, respectively (11). In study of Ruiz et al. in Ecuador, the prevalence of nasal carriage of MRSA among ICU staff was $2.4 \%$ (12). In some studies, the frequency of nasal carriage of $S$. aureus among hospital staff has been reported to be ranging between $11.9 \%$ and $33.28 \%$ (13-16). However, in a study in Iran, the prevalence of MRSA nasal carriage among a hospital's staff was $5.3 \%$ (17). Before the intervention, of the 120 ICU patients, only five patients had hospital infection caused by $S$. aureus. This highlights that the infection control in the ICU has been satisfactory. Moreover, S. aureus was completely eradicated after the treatment with mupirocin nasal ointment. Two similar studies found the eradication rate of mupirocin to be $89.5 \%$ and $83.5 \%(18,19)$. A study claimed that $91 \%$ eradication rate could be achieved with twice-daily administration of mupirocin for five days (20). Contrary to the previous studies, we completely eradicated nasal carriage of $S$. aureus using mupirocin ointment. The intervention was also successful in reducing the incidence of infection with $S$. aureus and other bacteria in the ICU. Of 120 patients admitted to the ICU during the threemonth assessment, 72 patients had at least one 
positive culture, while after the intervention, only 30 cases had positive culture results. This can be attributed to the greater hand hygiene adherence and better control of infection in the ICU. Our findings revealed that the prevalence of nasal carriage of $S$. aureus among ICU staff and patients was lower than that reported by other studies in Iran. Generally, the low prevalence of carriers in the ICU could be associated with the low number of nursing staff and the restrictions in this unit. Furthermore, no $S$. aureus was isolated after the treatment of the patients with mupirocin nasal ointment, indicating the high efficacy of the antibiotic in eradication of $S$. aureus carriage.

\section{CONCLUSION}

Compared to previous studies in Iran, the

\section{REFERENCES}

1. Silva EC, Antas Md, Monteiro B Neto A, Rabelo MA, Melo FL, et al. Prevalence and Risk Factors for Staphylococcus aureus in Health-Care Workers at a University Hospital of Recife-PE. Braz J Infect Dis. 2008; 12(6): 504-8.

2.Vinodhkumaradithyaa A, Uma A, Shirivasan M, Ananthalakshmi I, Nallasivam P, Thirumalaikolundusubramanian P. Nasal Carriage of Methicillin-Resistant Staphylococcus aureus among Surgical Unit Staff. Jpn J Infect Dis 2013; 62(3): 228-229.

3. de Carvalho MJ, Pimenta FC, Hayashida M, Gir E, da Silva AM, Barbosa CP, et al. Prevalence of methicillin-resistant and methicillin-susceptible $S$. aureus in the Saliva of health professionals. CLINICS. 2009; 64(4): 295-302.

4. Treakle A, Thom KA, Furuno P. Bacterial contamination of health care workers white coats. Am J Infect Control. 2009; 37(2): 101-105. doi: 10.1016/j.ajic.2008.03.009.

5. Bertman G, Katzung MD. Basic Pharmacology. NewYork: Mc Grow-Hill. 2016; 793-802.

6. Ojulong J. Mwambu TP, Jolobo M, Agwu E, Bwanga F, Najjuka C, et al. Prevalence of Methicillin Resistant Staphylococcus aureus (MRSA) Among Isolates from Surgical site Infections in Mulago Hospital, Kampala, Uganda. The Internet Journal of Infectious Disease. 2009; 7.

7. Saderi H, Owlia P, Maleki Z, Habibi M, Rahmati N. Susceptibility to Vancomycin in Staphylococcus aureus Isolated From Patients of Four University-Affiliated Hospitals in Tehran. Iranian Journal of Pathology. 2008; 3: 161-166.

8. Forbe BA, Sahm DF, Weissfeld AS. Bailey \& Scotts Diagnostic Microbiology. $14^{\text {th }}$ ed. Mosby, New York. 2015; 172-213.

9. CLSI: Clinical and Laboratory Standards Institute. Performance standards for antimicrobial susceptibility testing; $24^{\text {th }}$ informational supplement. CLSI, Wayne, PA. M100-S21 2017; 31(1). 8: 161-165.

10. Louie L, Goodfellow J, Mathiew P, Glatt A, Louie M, Simor A. Rapid detection of Methicillin resistant Staphylococci from blood culture bottles by using a multiplex PCR assay. J Clin Microbiol. 2002; 40(8): 2786-2790.

11. Rahimi-Alang S, Asmar M, Cheraghali F, Yazarlou S, Amini A, Shakeri F, et al. Frequency of methicillin resistant Staphylococcus aureus in healthcare workers in Gorgan. Zahedan J Res Med Sci (Tabib-e-shargh). 2011; 13(1): 17-22. [Persian]

12. Ruiz A, Mora M, Zurita C, Larco D, Toapanta Y, Zurita J. Prevalence of methicillin-resistant Staphylococcus aureus prevalence of $S$. aureus nasal carriage is relatively low among the ICU staff and the patients of the Panje-azar hospital in Gorgan, Iran.

Our findings also demonstrate that mupirocin nasal ointment is effective in eradication of $S$. aureus carriage and subsequently contribute to reduction in frequency of nosocomial infections.

\section{ACKNOWLEDGMENTS}

The study has been financially supported by the Infectious Disease and Medical Laboratory Sciences Research Centers of the Golestan University of Medical Sciences, Iran.

\section{CONFLICT OF INTEREST}

The authors declare no conflict of interest.

among health care workers of intensive care units in Ecuador. $\mathrm{J}$ Infect DevCtries. 2014; 8(1): 116-9.

13. Khalili M, Moshref M, Sharifi M, Sadeh M, Sazmand A. Prevalence of Staphylococcus aureus (SA) and Methicillin resistant Staphylococcus aureus (MRSA) in personnel of Operation room of Shahid Sadoughi hospital, Yazd, Iran. Payavard Salamat. 2013; 6(5): 392-402. [Persian]

14. Shibabaw A, Abebe T, Mihret A. Nasal carriage rate of methicillin resistant Staphylococcus aureus among dessie referral hospital health care workers; dessie, Northeast Ethiopia. Antimicrob Resist Infect Control. 2013; 2: 25. doi: $10.1186 / 2047-2994-2-25$

15. Serag-Eldin AS, Amany TA, Noha GA, Mohamed EAA. Clinical and microbiological effect of linezolid on Methicillin resistant Staphylococcus aureus (MRSA)colonization inhealthcare workers in Egypt. Middle East J Sci Res. 2013; 15(10): 1440-9.

16. Mansouri Ghiasi MA, Nasrollahi Omran A, Hashemi M, RajabZade Kanafi P, Jahangiri Rad M. The prevalence of antibiotic resistance pattern of Staphylococcus aureus isolatedfrom nasal carriage of surgical ward's staff in Shahid Rajaee hospital of Tonekabon, Iran. Medical Laboratory Journal. 2013; 7(1): 35-39. [Persian]

17. Askarian M. Zeinalzadeh A, Japoni A, Alborzi A, Memish ZA. Prevalence of nasal carriage of methicillin-resistant Staphylococcus aureus and its antibiotic susceptibility pattern in healthcare workers at Namazi Hospital, Shiraz, Iran. International Journal of Infectious Diseases. 2009; 13(5):e241-7. doi: 10.1016/j.ijid.2008.11.026

18. Sarmadian, h. Dydgr, f. Abtahi, h.2008. Comparison of mupirocin nasal cream effect of single-dose oral ciprofloxacin in the treatment of recurrent nasal carriers of Staphylococcus aureus in personnel in Valiasre. Journal of Medical Sciences, 2004: Vol. 11, No. 1, pp, 40-44.

19. Kalmeijer MD, Coertjens H, van Nieuwland-Bollen PM, Bogaers-Hofman D, de Baere GA, Stuurman A, et al. Surgical Site Infections in Orthopedic Surgery: The Effect of Mupirocin Nasal Ointment in a Double-Blind, Randomized, PlaceboControlled Study. Clin Infect Dis. 2002; 35(4): 353-8.

20. Doebbeling BN, Breneman DL, Neu HC, Aly R, Yangco BG, Holley HP Jr, et al. Elimination of S. aureus nasal carriage in health care workers: analysis of six clinical trials with calcium mupirocin ointment. Clin Infect Dis. 1993; 17(3): 466-74. 